\title{
Future Power Grid Dispatch and Control Mode with Large-scale Clean Energy Integration in China
}

\author{
Cai Zhi ${ }^{1}$,, Xu Dan ${ }^{1}$, Dai Sai ${ }^{1}$, Cui Hui ${ }^{1}$, Ding Qiang ${ }^{1}$ \\ ${ }^{1}$ China Electric Power Research Institute, Beijing, China \\ acaizhi@epri.sgcc.com.cn
}

\begin{abstract}
Keywords: Future power grid; Dispatch mode; Control mode; Power system
\end{abstract}
Abstract. Smart grid is the development trend of power system in China. Intelligent dispatch and control system, as an important part of smart grid, is the new development direction of future automation system. It is of significant necessity to study on future dispatch and control mode. This paper reviews the evolution of power grid dispatch and control mode and analyzes the major influence factors and key technologies for its development. A concept design of future grid dispatch and control mode is proposed, which adapts to the construction of smart grid with large-scale clean energy integration, and provides more comprehensive and intelligent support for operators.

\section{Introduction}

At present, China's power grid dispatch system mainly includes all levels of dispatch departments, substation operational units, and power plants. The operation and coordination in the power system involves different voltage levels grid, the main grid and distribution network, centralized and distributed generation. They follow the principles in the business: substation operational units and power plants must obey the dispatch departments, while the lower dispatch departments must obey superiors. In general, the primary objective of the current power system in China is to ensure the safety and stable operation, while the economy, environmental protection and other control targets are relatively ignored.

\section{Factors for the future grid dispatch and control mode}

Under the pressure of global environmental and resource problem, energy-saving and emission-reduction has become the most important requirement to meet in the future. On the one hand, the loss of resources should be reduced; on the other hand we want to change the composition of power resources. This means the power generated by fossil energy share in total energy should be reduced, thus clean renewable energy such as wind energy, solar energy, tidal energy, geothermal power generation proportion should be greatly increased.

In the generation side, due to the requirements of environmental and economic factors, a large-scale renewable energy based and fossil energy supplemented pattern will be formed. The centrally located power centers and loads do not coincide, requiring large-scale renewable energy through high-capacity, high-voltage, long-distance power transmission, using a variety of new, flexible transmission technology connected to the grid. In addition, most renewable power plants connected to the grid by power electronic devices, in order to improve the grid power quality after the transforming process. Integration of energy storage systems enhance the stability of the grid and accelerate the outage recovery.

Compared to the generation side, there will be more significant changes in demand side in the future. Demand-side equipment will be greatly expanded, distributed power, electric vehicles, energy storage and other power electronic converter will connect to the grid, so that the structure of the distribution network and energy flows will be more complex and flexible. In most areas, these devices pose a micro-grid, which can be switched off-grid or on-grid state to ensure continuous power supply to the load and reduce the scope of the power grid failures and provide power to support the grid. Intelligent 
power distribution network equipment, which can flexibly adjust power needs, help maintain the grid power balance. This provides the technical support for the realization of the demand-side response.

\section{Dispatch mode adaptability analysis}

The adaptability of dispatch mode mostly depends on whether it is adapt to the development of industrial under high proportion of clean energy power, the specific analysis can be concluded as follow:

(1) wide range of optimal allocation of resources

The rapid development of enhanced economic integration in today's power industry and other related industries, especially with the coal industry and other energy resources is inextricably linked. In the energy resource depletion, concerns about the deteriorating natural environment background, how to make effective energy resources, produce the greatest benefits becomes a hot issue. Therefore, advanced dispatch mode, should be able to achieve the optimal allocation of resources from a larger ranger, in many types of energy (including conventional thermal power, wind power, photovoltaic, pumped storage, energy storage and demand-side load, etc.) Due to the different characteristics of various power generating units, security, economic, energy and environmental factors such as power system operation should be considered to meet the various requirements of complex constraints random factors from intermittent energy, energy efficiency should be improved. Multi-energy has a greater complementary optimization potential. The implementation of a Multi-energy complementary scheduling, can utilize all kinds of power expertise, complementary advantages, not only can promote wind power and photovoltaic consumptive, support new energy development, and reduce the energy-saving emission, but also improve the quality of supply, and guarantee the security and stability of economic operation. At the same time, a wide range of optimal allocation of resources can improve the level of intermittent energy consumption; reduce the impact of clean energy uncertainty, randomness and anti-peaking on the system safety and stable operation.

(2) system security requirements

Power system security is a characteristic manifested during operation, which is the ability of continuously providing users with stable supply under disturbances. Given the importance of the power system in the national economy and social life, countries have developed power system security and stability standards respectively, which ensure the safety of the system as a prerequisite for system operation. Thus, whether the dispatch mode is advanced, the most important thing is whether the operation mode is conducive to the improvement of security of the system. The requirements of safety evaluation system can be summed up to follows: higher capacity to withstand accidents, accurate load forecasting ability, the ability to secure power supply, control capabilities, the correct rate of scheduling and execution of instructions, accident response ability.

(3) economic operation

The nature of public utilities power system, determines the electric power system operation should meet social benefits; at the same time, nature of the company's electricity business, determines its basic goal is the pursuit of profit. From both points of view, the pursuit of efficiency is a core objective of power system operation, therefore, a way to maintain and to run the system development, will be able to realize sufficient economy under the premise of safe operation.

(4) adaptation to technological development

A variety of renewable energy resources has been the rapid developed in recent years and power grid moves in intelligent direction, thus, power system operation faces many new challenges. Whether the new operating system can respond to these challenges and adapt the latest power technology developments, is another key factor to judge dispatch mode adaptation.

\section{Control mode adaptability analysis}

In the development process of power grid and its control system, the power control technology has experienced a long-term history from simple to complex and from lower to higher. With the development of technology, a variety of new forms of power generation and consumption will emerge and the control requirements will be more diverse and more complex. Roughly speaking, the grid control requirements can be divided into external demand and internal demand. From the internal grid 
view, the requirements for the control objectives include the safety and economy; from the outside, the requirements of the control objectives include power quality, environmental protection, interactivity and compatibility.

In order to meet these requirements, the future control performance will have characteristics as follows:

(1) optimization

The Optimization in the economy includes: low net loss, high capacity utilization, low expense control; in power quality: voltage quality, small voltage fluctuation, frequency high pass rate; in reliability: power supply reliability, low annual average outage time; in environmental: fewer emissions, low energy loss; in user experience: customer satisfaction, feedback evaluation.

(2) robustness

Control system design will maintain good control performance under different situations such as various operation states, failures, load fluctuations. There will be a large number of users take interactive participation in electrical grid in the future. The grid may even be able to obtain communications and Internet features, then, the control of the future grid should be able to cope with interference entities to preserve robustness.

(3) adaptability

Adaptability means that the control law doesn't need to redesign when the model parameters or the state of the grid changes. In addition, the accommodation control of new energy and flexible load should also be self-adaptive. The control mode will be adaptive to various power generating units and electrical equipment type.

(4) intelligence

The future grid will behave like a intelligent robot, which have monitoring, evaluation, event analysis and decision-making, and a series of mechanisms to achieve a high degree of artificial intelligence, such as automatic identification, recovery and self-healing function. Intelligence of future grid will be also reflected in the large number of intelligent control and electrical equipment for customer services. In the demand-side view, the function is not only to provide the power supply grid, but also be capable of making an appropriate response to instructions and providing power optimization, household equipment management and information services.

\section{Concept design}

Future grid business model includes both centralized and distributed energy, both actively response and fixed demand, both centralized scheduling and covert dispatching, covering the power generation / transmission / distribution /industry chain / fair competition systems.

In power generating, centralized and distributed power will participate in the market freely.

In energy consuming, fixed load and demand response can participate in different levels markets in accordance with $t$ the different types of resources, to seek their own interests.

In power dispatch department, dispatch center centralized scheduling, while energy storage systems and demand response make use of market signals to participate implicitly.

Coordination among power system optimization modes includes hierarchical partitioning autonomous coordinated dispatching, multi-level coordination of reactive power control, situational awareness and risk prevention. Multi-period optimized scheduling modes include centralized and distributed multi-level coordination, multi-period multi-resource coordination and electricity market and so on.

\section{future grid dispatch mode}

Expected future grid dispatch mode with high proportion of clean energy will be "centrally coordinated, local self-government". The wide-area cooperative operation control architecture includes independent and robust dispatch operation mechanism. Future grid will form a layered coordinated operation autonomous dispatch and coordination of multi-level reactive power control. 
The transmission network, distribution network, and micro-grid hierarchical control system optimize scheduling to achieve a coordinated dispatch between different periods.

In large grid, the flow of information is enormous. If the information are processing, analysis and transmission by the same dispatch center, not only uneconomic, but also not conducive to rapid response. In addition, because the most important scheduling decisions are still done by people, the dispatchers of a dispatch center will not be enough in the face of such a large grid. A hierarchical grid will be need, and if a regional power grid is still relatively large, it can be further subdivided. Dispatch scales at each level of the process are not same. More by the upper layer of the dispatch center, its scheduling is broader, more macro-grid areas. Closer to the lower, the narrower range of scheduling, scheduling work will be more sophisticated. Lower dispatch center obeys the command from upper dispatch center, which includes active and reactive power regulation, load forecasting, economic dispatch, maintenance plan making, real-time warning and management, security check and so on. The main features of the future dispatch mode are as follows:

(1) Multi-period multi-resource optimization will coordinate scheduling objectives assigned to the layer. The difference between actual power and predicted value can be adjusted by demand-side response to achieve power balancing. Upper communications support and distributed processing mode, changing the traditional information collection and centralized processing pattern, will greatly increase the flexibility and complexity of the future grid information flows. Due to the improvement of power quality and stability, the dispatching will focus on guiding and controlling the energy flow of the entire system, for the optimized allocation of social resources and improving energy efficiency.

(2) Energy storage devices can store up fossil, hydro and chemical energy as a reserve capacity of the system. With the applications of Energy storage device in the power grid, the balance of power doesn't need to be achieved instantaneous. By energy storage device charging and discharging operations, some excess energy can be stored and released in other periods, which means that the energy of the grid can be flexibly flows across time. When the system fails or power balance cannot be met simply by power adjustment, these devices release or store energy, to ensure the system's power balance and maintain security and stability of the system.

(3) Distributed generation, smart electrical equipment and demand side of the energy storage device consist of micro-grid, which can achieve a local balance of power, improve power efficiency and reduce the scope of grid failure. Micro-grid technology will change the radial supply patterns and build more controllable flow channels among distribution network and micro-grids to realize flexible energy flows.

(4) Based on the condition of the grid, demand-side response can control or adjust the load power scheduling. It can realize load shifting to improve the overall operational efficiency and ensure grid security and stability in case of failure. Demand response will extend the grid to the endings, which is able to provide information and accept the dispatch instructions. To maintain balancing, demand response will be utilized to establish a two-way information flow between the dispatch center and distributed demand side, which includes demand-side micro-grid internal scheduling, dispatching between the micro-grids and direct load dispatch. In the micro-grids, information will be produced and released through some distributed automatic facilities, while the manual dispatch will only need to focus on the external characteristics of these micro-grids on the upper level of the electricity network.

\section{future grid operational control mode}

Future grid operational control will be AC-DC multi-level optimization of active and reactive coordinated control. Price incentives and load shifting will be effectively used to mobilize the power side and the demand side to form a interactive control in transmission network, distribution network and micro-grid energy management systems.

At present, the underlying general closed-loop control is local and device-level; the middle level is generally closed-loop system-level control. Up to a certain level of control is open-loop, which can be considered closed-loop control with the manual participation. The future trend is the top of the closed-loop control is fully automatic. 
In the coming decades, the control mode will made significant progress. These innovations will be mainly due to the development of grid architecture changes, and the development of control technology will assist this progress. The main features of the future grid control system will be still the automation and intelligence, because regardless of the control system development, its ultimate goal is to replace people to achieve the set goals, and its performance in implementing scheduled tasks will reflect its intelligence.

(1) Combining centralization and decentralization is the principle of grid control and will have an updated content in the future. The traditional power grid emphasizes on centralized optimization, and decentralized control mostly focuses on information acquisition. Future grid control trends are contrary, which are more emphasis on the individual, decentralized, local control unit autonomy and intelligence, trying to solve as many problems in local processing as possible, so that the local control unit will not act as a subsidiary unit, but as an autonomous system to automatically complete control and operation functions.

However, this is not to abandon the centralized control, but to a higher level of coordination so as to form a hierarchy, autonomy and synergy characterized by distributed control architecture.

(2) In the future, information and communication and computer technology will provide favorable conditions for the global information acquisition and processing. PMU and wide-area measurement system will be further developed, a comprehensive wide area measurement system of the whole system is likely to be established, in addition, wireless broadband network technology may also be applied to the power supply control. These technologies development form the foundation for the wide area control system. In order to realize the future local and global optimization target control, more and more effective real-time wide-area information must be utilized to achieve local and wide-area coordinated control system.

(3) Future grid control system should be fault-tolerant and self-healing, so that self-organized control system is one of the feasible way. Self-organized control system is capable of evolving or improving its structure in interaction with environmental conditions. In the future grid, system-level control system will coordinate lower levels according to the environment and their own operation situation to form the best organizational structure, which can automatically fix defects and faults, restore normal structure and function. Self-organized control system for improving the robustness of future grid has important significance.

\section{Conclusions}

Power is the foundation of the national economy, which concerns national energy security and social development. Evolution of power grid operation form is a long historical process, which is driven by many factors. The evolution of these factors will set forth more requirements in the future. Under a high proportion of clean energy, flow of energy and information will be more flexible and complex, which rely on energy storage technologies, micro-grid technology, demand response and other key technology support. From the technologies and their development, it can be predicted that the future power grid will be formed "centralized coordination, local autonomy", forming transmission network, distribution network, micro-grid energy management systems interactive dispatch and control system.

\section{Acknowledgements}

This work was financially supported by China Electric Power Research Institute.

\section{References}

[1] Alex Huang - FREEDM system - a vision for the future grid [A] . 2010 IEEE Power and Energy Society General Meeting [C] . Minneapolis , Minnesota , USA , 2010. 1-4 . 
[2] Ipakchi A , Albuyeh F . Grid of the future [J] . IEEE Power and Energy Magazine , 2009 , 7 ( 2) : 52-62 .

[3] Monti A , Ponci F . Power grids of the future: why smart means complex [A] . 2010 Complexity in Engineering[C] . Rome, Italy, 2010. 7-11.

[4] He Haibo . Toward a smart grid: integration of computational intelligence into power grid [A] . The 2010 International Joint Conference on Neural Networks [C] . Barcelona , Spain , 2010. 1-6 .

[5] Enrique Santacana, Gary Rackliffe, Le Tang, et al . Getting smart [J] . IEEE Power and Energy Magazine , 2010 , 8 ( 2) : 41-48 .

[6] Ahshan R , Iqbal M T , Mann G K I , et al . Micro-grid system based on renewable power generation units [A] . 23rd Canadian Conference on Electrical and Computer Engineering [C] . Calgary Alberta , Canada , 2010 .

[7] Siemens A G . Advanced architectures and control concepts for more microgrids [R] . Erlangen , Germany , 2009.

[8] Amir-Hamed Mohsenian-Rad, Vincent W S Wong, Juri Jatskevich, et al . Autonomous demand-side management based on game-theoretic energy consumption scheduling for the future smart grid [J] . IEEE Transactions on Smart Grid , 2010 , 1 ( 3) : 320-331 .

[9] Peter Palensky, Dietmar Dietrich . Demand side management: demand response, intelligent energy systems , and smart loads [J] . IEEE Transactions on Industrial Informatics , 2011,7 ( 3) : 381-388. 